\title{
Análisis del material del habitáculo de seguridad de un vehículo liviano por método multicriterio
}

\section{(Analysis of the safety compartment material of a light vehicle by multi-criteria method)}

\author{
Edwin Chamba, ${ }^{1},{ }^{2}$ Llanes Cedeño Edilberto Antonio, ${ }^{1}$ \\ Andrés Cárdenas-Yánez, ${ }^{1,3}$ William Vega, ${ }^{1}$ Rocha Hoyos Juan Carlos, ${ }^{1,4}$
}

\begin{abstract}
Resumen
Existe una gran variedad de materiales disponibles que cuentan con diversas propiedades para que los diseñadores satisfagan los diferentes requerimientos de diseño. El presente estudio tuvo como objetivo evaluar las propiedades de los materiales relevantes disponible en el Ecuador a través de la aplicación del método de criterios como son: evaluación compleja proporcional (COPRAS), técnica para el orden de preferencia por similitud a solución real (TOPSIS), optimización multidisciplinar y solución de compromiso (VIKOR), con el fin de indicar cuál material sería un potencial sustituto del original. Se concluye que el acero 1045 es la mejor opción como sustituto para un habitáculo de seguridad, mientras que la aleación Aluminio-Magnesio, a pesar de ser un material ligero posee baja resistencia a las fuerzas de impacto.
\end{abstract}

\section{Palabras clave}

Habitáculo de seguridad, método multicriterio, optimización, selección de materiales, MCDM, vehículo

\begin{abstract}
There is a wide variety of materials with various properties available for designers to meet different design requirements. The objective of this study was to evaluate the properties of the relevant materials available in Ecuador through the multicriteria meth-od such as: Proportional complex evaluation (COP-RAS), Technique for the order of preference for similarity to real solution (TOPSIS), Optimization multi-disciplinary and compromise solution (VIKOR), in order to indicate which material would be a potential substitute for the original. It was concluded that 1045 steel is the best choice as a substitute for a safety compartment compared the base line the Aluminum-Magnesium alloy, considering that, despite being a light material, has low resistance to impact forces.
\end{abstract}

\section{Keywords}

Security cabin, Multi-criteria method, Optimization, Materials selection, MCDM, vehicle.

\section{Introducción}

El rápido desarrollo de la economía global y de la tecnología de ingeniería ha permitido que la industria del automóvil evolucione rápidamente, lo que ha revolucionado el estilo de vida de las personas en las últimas décadas, uno de los sectores es el automotriz, por lo que el uso de vehículos se ha vuelto de consumo popular. Sin embargo, los accidentes de tráfico se han convertido simultáneamente en un grave problema social. La mortalidad causada por el accidente del vehículo se ha mantenido en un alto nivel. El impacto frontal representa el $40 \%$ de todas las Industria Automotriz Ecuatoriana. Quito-Ecuador (echamba.mdm@uisek.edu.ec, antonio.llanes@uisek.edu.ec, acardenas@ uisek.edu.ec, wvega.mdm@uisek.edu.ec, carlos.rocha@uisek.edu.ec)

2 Corporación de Electricidad del Ecuador (CELEC EP), Quito-Ecuador

3 Instituto Superior Tecnológico Cotopaxi, Latacunga-Ecuador

4 Escuela Superior Politécnica de Chimborazo, Facultad de Mecánica, Carrera de Ingeniería Automotriz, Riobamba-Ecuador (juan.rocha@espoch.edu.ec). 
colisiones de vehículos, causando el 70 \% del total de muertes de tráfico (Wang, y Wang, 2016). En un informe emitido por la Organización Mundial de la Salud (OMS), en el año 2010 se reporta al Ecuador como uno de los países con más número de muertes por accidentes de tránsito en Latinoamérica. El 50.09 \% son ocasionados por la falta de experiencia o descuido del conductor, el 13.2 \% por desacato a las leyes de tránsito, el 12.31\% por superar los límites de velocidad permitidos, otro factor es la embriaguez con un $9.73 \%$, el 7,69 \% por situaciones externas sin comprobar y el $6.99 \%$ por descuido de otros implica-dos (ANT, 2015). Se considera que una prueba de choque es una forma de pruebas destructiva para garantizar un diseño seguro en caso de impacto o colisión. La simulación de choque es muy aplicada últimamente, porque es una representación virtual de un ensayo destructiva de impacto de un vehículo empleando una simulación por computadora con el fin de examinar el nivel de seguridad del vehículo y sus ocupantes en diferentes condiciones como la velocidad de colisión, colisionar el objeto, el material y el número del componente del objeto colisionando, entre otros, por lo tanto, ahorra el tiempo y el costo (Mishra y Pradhan, 2016; Villarruel-Jaramillo et al., 2019).

El Ecuador se encuentra innovando de manera constante y se actualiza de acuerdo a las normas de seguridad vehicular, el primer paso fue la emisión y cumplimiento del Reglamento Técnico Ecuatoriano (RTE) 034, Anexo III A, que exigió la incorporación de elementos mínimos de seguridad en cualesquiera de los automóviles que se comercialicen (Arízaga y Gómez, 2015; Rocha-Hoyos et al., 2017). La industria ensambladora y fabricadores de autopartes del Ecuador ha realizado grandes inversiones para el cumplimiento de la normativa. En la actualidad, el resultado es alentador, ya que cualquier vehículo que se ensamble o fabrique en el país cuenta con estándares de seguridad que van a la evolución con los países de la región.

La Figura 1 muestra la viga longitudinal delantera (FLB) que tiene un patrón mixto de deformación axial y de flexión bajo el impacto frontal del vehículo. En comparación con la deformación por flexión, el modo de deformación axial es un patrón preferido para absorber la energía cinética. Para aprovechar al máximo el espacio de trituración de la zona de aplastamiento y explotar el máximo potencial de absorción de energía (Duan et al., 2016).

Figura 1. Distribución de la trayectoria de carga en el impacto frontal (Duan et al., 2016)

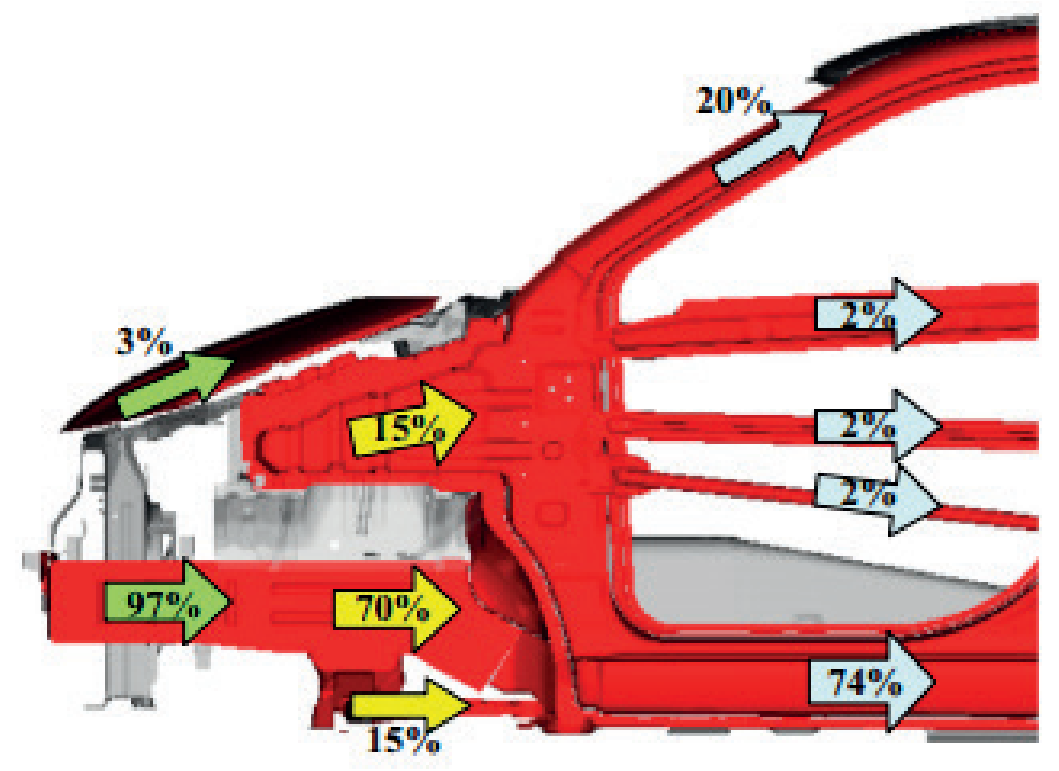


Para seleccionar la mejor alternativa para el presente estudio, se evaluarán 7 propiedades de los materiales relevantes; se utilizarán los siguientes métodos para tomar decisión de cuál es el material más apropiado (Zafra-Cabeza et al., 2019).

- Evaluación compleja proporcional (CO-PRAS).

- Técnica para el orden de preferencia por similitud a solución real (TOPSIS).

- Optimización multidisciplinar y solución de compromiso (VIKOR).

El presente estudio tendrá como objetivo evaluar las propiedades de los materiales relevantes disponible en el Ecuador a través de método de multicriterios para la selección del material potencial sustituto del original.

\section{Metodología}

En la presente investigación se aplicó la técnica de investigación que es un procedimiento algorítmico (Latorre y Seco, 2013; Vega, Llanes, Molina, Rocha-Hoyos, 2018), que dará forma a la realización de la investigación planteada dividida en tres partes, con pasos lógicos y ordenados a seguir para concretar el estudio (Guardia, Márquez, Sánchez, Llanes-Cedeño, Rocha-Hoyos y Peralta, 2018; Llanes-Cedeño, Rocha-Hoyos, Peralta, Martínez y Celi, 2018). Al respecto de su alcance es del tipo experimental, pues evalúa las variables de forma controlada.

Se realizó el ensayo de tracción, que consiste en someter a una probeta normalizada del material a un esfuerzo de tracción hasta que se produzca la rotura, este ensayo mide la resistencia de un material a una fuerza aplicada lentamente. Las velocidades de deformación en un ensayo de tensión deben ser pequeñas; ya que pueden determinarse diversas características de los materiales elásticos: Módulo de elasticidad o Módulo de Young, Coeficiente de Poisson, Límite de proporcionalidad, Límite de fluencia o límite elástico aparente, Límite elástico, Carga de rotura, Alargamiento de rotura, Área de estricción.

Para obtener las características mecánicas reales, se realizaron 5 probetas bajo la norma técnica ASTM E8 del material del habitáculo de seguridad a pruebas de tracción como se observa en la figura 2 (Rocha-Hoyos et al., 2019), las pruebas se realizaron en el laboratorio de análisis de materiales del centro de Fomento Productivo carrocero Metal Mecánico de la ciudad de Ambato, obteniéndose los resultados mostrados en la tabla 1.

Tabla 1. Resultados de caracterización de materiales

\begin{tabular}{|l|l|l|}
\hline \multicolumn{1}{|c|}{ Probeta } & \multicolumn{1}{|c|}{ Resistencia a la rotura (MPa) } & \multicolumn{1}{c|}{ Límite de fluencia (MPa) } \\
\hline 1 & 197.25 & 195.09 \\
\hline 2 & 139.06 & 139.73 \\
\hline 3 & 219.33 & 202.06 \\
\hline 4 & 238.94 & 230.15 \\
\hline 5 & 201.09 & 205.16 \\
\hline Promedio $\frac{\dot{x}}{}$ & 247.234 & 194.438 \\
\hline
\end{tabular}


Figura 2. Probetas del material

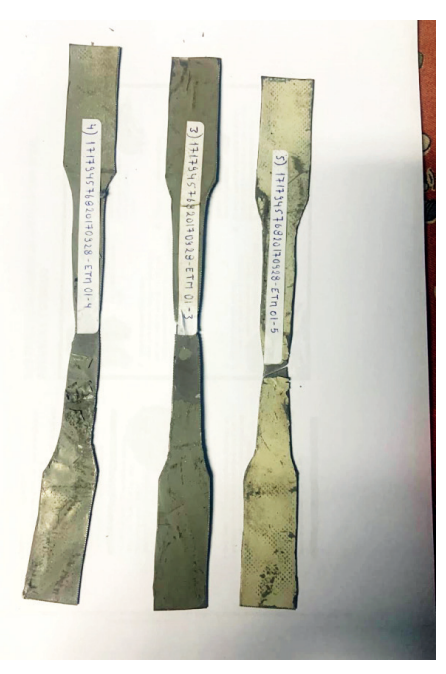

La figura 3, muestra información proporcionada de la caracterización del material, mediante el esfuerzo de deformación. La prueba de tracción permitió obtener el patrón para de la curva de esfuerzo-deformación en las 5 probetas experimentadas.

Figura 3. Gráficos del ensayo de tracción de materiales

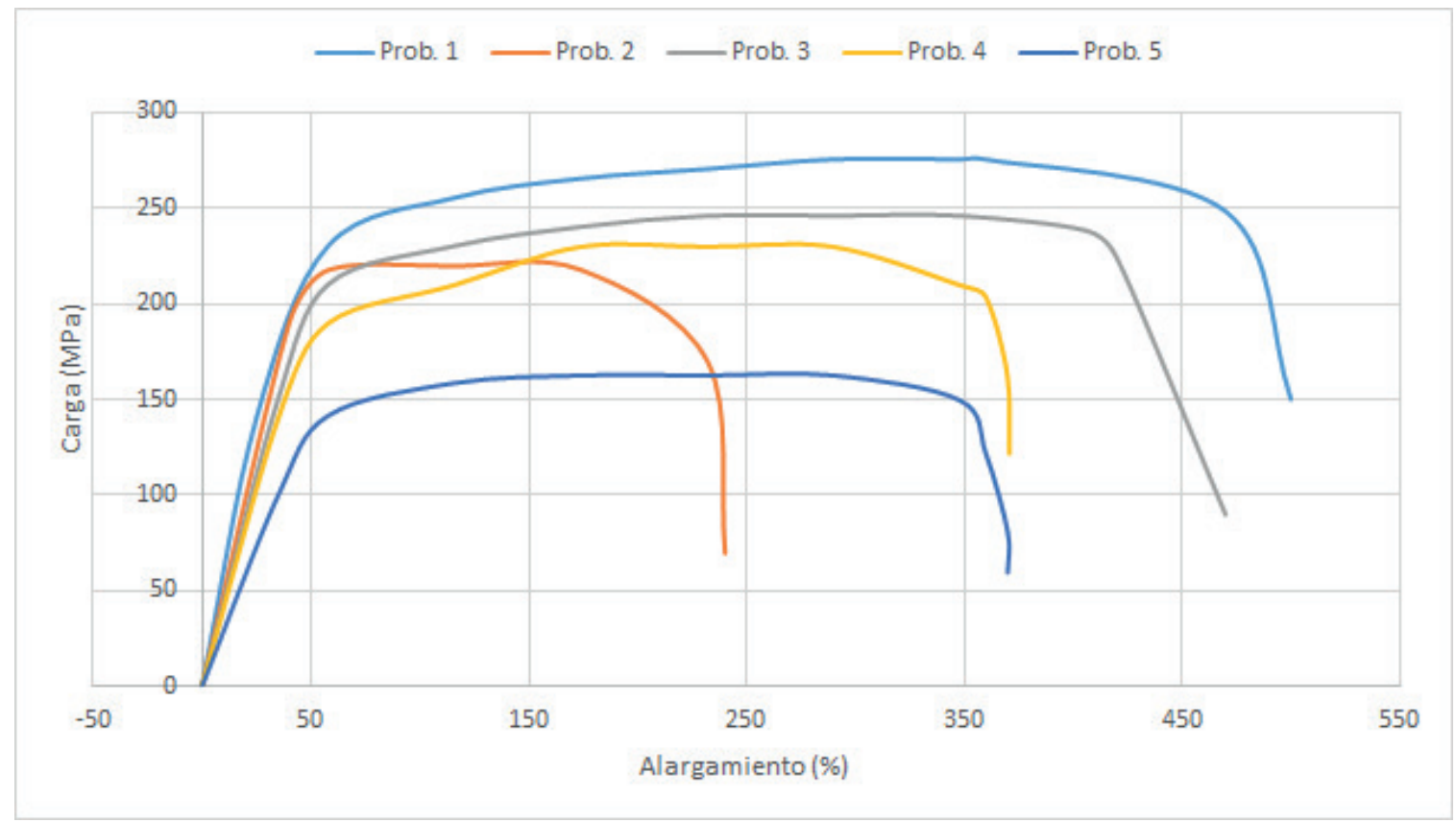

Para seleccionar la mejor alternativa, se evalúan 7 propiedades de los materiales más relevantes presentes en el mercado ecuatoriano, como se presenta en la tabla 2. Se utilizaron métodos multicriterio para la selección del material más apropiado, dichos métodos son: 1) Evaluación compleja proporcional (COPRAS). 2) Técnica para el orden de preferencia por similitud a solución real (TOPSIS). 3) Optimización multidisciplinar y solución de compromiso (VIKOR) (Ashby y Johnson, 2010; Chérrez-Troya et al., 2018). 
Tabla 2. Propiedades de materiales

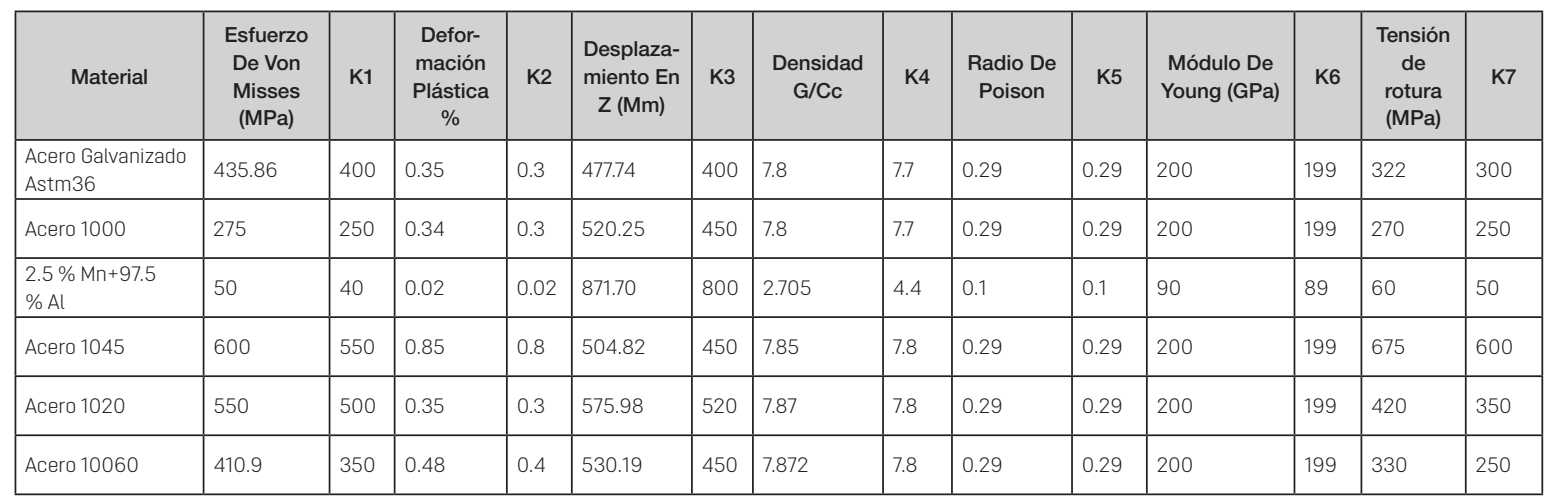

\section{Resultados y discusión}

\subsection{Método COPRAS}

Evalúa las alternativas realizando el sumatorio del valor normalizado de cada criterio por su peso correspondiente. Puede ser aplicado tanto para criterios que se desean maximizar como minimizar (Pla, 2017). Como primera variable es necesaria la construcción de una matriz inicial $X$, que permitirá anticipar las alternativas para la selección de criterios, como se muestra en la tabla 2.

Un problema común es la selección de criterios al diseñar la matriz, ya que algunas no presentan iguales dimensiones en filas y columnas, para superar la matriz X, es modificada por una matriz adimensional r como se indica en la ecuación 1.

$$
r_{i j}=\sqrt[x_{i j}]{\sum_{i-j}^{m} x_{i j}^{2}} \quad i=1, \ldots, m_{i} \mathrm{j}=1, \ldots, \mathrm{n}
$$

El cálculo de la matriz de peso normalizada, mediante ponderaciones de la forma Vij de acuerdo a la ecuación 2 y 3 (Kundakci, y Işik, 2016).

$$
V_{i j}=r_{i j} x w_{j} \quad i=1, \ldots, m ; j=1, \ldots, n
$$

Donde $w_{j}$ es el peso de todos los $j^{\text {ésimos }}$ Criterios o atributos de acuerdo.

$$
r_{i j}=\sum_{j=1}^{n} \quad w j=1
$$

Se continúa, mediante la determinación de la matriz de peso normalizada D, es decir la suma de los valores normalizados de peso adimensionales, en cada criterio, es siempre igual al peso como cada criterio, según ecuación 4.

$$
D=\left[y_{i j}\right] r_{i j}{ }^{*} w_{j}
$$

Donde $r_{i j}$ es el valor normalizado de la $i$ ésimos alternativa sobre el $j$ ésimos criterio y $w_{j}$ es el peso del $j$ ésimo criterio. Como último paso se realiza la sumatoria de atributos de la matriz de decisión, es decir, atributos beneficiosos y no beneficiosos, de acuerdo al criterio de evaluación, 
es decir son asociados una maximización o minimización, de acuerdo a la ecuación 5, esto se refleja como resultado en la tabla 3.

$$
S_{+i}=\sum_{i=1}^{m} \quad y+i j
$$

Tabla 3. Método COPRAS - resultados

\begin{tabular}{|l|l|l|l|l|l|}
\hline \multicolumn{1}{|c|}{ Material } & \multicolumn{1}{c|}{$\mathbf{P i}$} & \multicolumn{1}{c|}{ Ri } & \multicolumn{1}{c|}{ Qi } & \multicolumn{1}{c|}{$\begin{array}{c}\text { PUNTUACIÓN } \\
\text { FINAL }\end{array}$} & RANGO \\
\hline ACERO GALVANIZADO ASTM36 & 0.1963 & 0.0004 & 0.1967 & 98.2696 & 3.0000 \\
\hline ACERO 1000 & 0.1874 & 0.0005 & 0.1879 & 93.8743 & 2.0000 \\
\hline $2.5 \% \mathrm{Mn}+97.5 \% \mathrm{Al}$ & 0.1594 & 0.0005 & 0.1599 & 79.8757 & 1.0000 \\
\hline ACERO 1045 & 0.2321 & 0.0006 & 0.2327 & 116.2756 & 6.0000 \\
\hline ACERO 1020 & 0.2231 & 0.0006 & 0.2237 & 111.7622 & 5.0000 \\
\hline ACERO 1006 & 0.1996 & 0.0005 & 0.2001 & 100.0000 & 4.0000 \\
\hline
\end{tabular}

\subsection{Método TOPSIS}

Según Pla (2017) dice que el método TOPSIS consiste en buscar soluciones que estén más cerca de la solución ideal positiva (SIP) y más lejos de la solución ideal negativa (NIP). El primer paso es formar la matriz decisión $n_{i j}$ de acuerdo a la siguiente ecuación 6 .

$$
n_{i j}=\frac{x_{i j}}{\sqrt{\sum_{i=1}^{n} x_{i j}^{2}}}
$$

Posterior se sincroniza el peso $w_{j}$ y se normaliza la matriz $n_{i j}$, con base en la siguiente ecuación 7, como paso 2.

$$
v_{i j=n_{i}}{ }^{*} w_{j} \quad(i=1,2, \ldots, m ; j=1,2, \ldots, n)
$$

Como paso 3, es necesario obtener soluciones positivas que se llamarán $\left(V^{+}\right)$y soluciones negativas $\left(V^{-}\right)$, para esto se conforma la matriz normalizada. Para lo cual se emplea las ecuaciones 8 y 9 :

$$
\begin{aligned}
& \left\{V_{1}^{+}, V_{2}^{+}, \ldots, V_{n}^{+}\right\}=\left\{(j \in K),\left(j \in K^{\prime}\right)\right\}\{i=1,2, \ldots, m\} \\
& \left\{V_{1}^{-}, V_{2}^{-}, \ldots, V_{n}^{-}\right\}=\left\{(j \in K),\left(j \in K^{\prime}\right)\right\}\{i=1,2, \ldots, m\}\{i=1,2, \ldots, m\}
\end{aligned}
$$

Donde $\mathrm{K}$ y $K^{\prime}$ son el conjunto de índices de los criterios de prestaciones y el índice de un conjunto de criterios en razón al peso. La tabla 4, indica los resultados de matriz con criterios positivos. La tabla 5, indica los resultados de matriz con criterios negativos. 
Tabla 4. Método TOPSIS-Matriz positiva

\begin{tabular}{|l|l|l|l|l|l|}
\hline \multicolumn{7}{|c|}{ MATRIZ POSITIVA } \\
\hline 0.12758128 & 0.04520412 & 0.14488235 & 0.13856208 & 0.04331492 & 0.03413313 \\
\hline 0.12758128 & 0.04520412 & 0.14488235 & 0.13856208 & 0.04331492 & 0.03413313 \\
\hline 0.12758128 & 0.04520412 & 0.14488235 & 0.13856208 & 0.04331492 & 0.03413313 \\
\hline 0.12758128 & 0.04520412 & 0.14488235 & 0.13856208 & 0.04331492 & 0.03413313 \\
\hline 0.12758128 & 0.04520412 & 0.14488235 & 0.13856208 & 0.04331492 & 0.03413313 \\
\hline 0.12758128 & 0.04520412 & 0.14488235 & 0.13856208 & 0.04331492 & 0.03413313 \\
\hline
\end{tabular}

Tabla 5. Método TOPSIS-Matriz negativa

\begin{tabular}{|l|l|l|l|l|l|}
\hline \multicolumn{7}{|c|}{ MATRIZ NEGATIVA } \\
\hline 0.05741157 & 0.00376701 & 0.01287843 & 0.00374945 & 0.01493618 & 0.06051712 \\
\hline 0.05741157 & 0.00376701 & 0.01287843 & 000374945 & 0.01493618 & 0.06051712 \\
\hline 0.05741157 & 0.00376701 & 0.01287843 & 0.00374945 & 0.01493618 & 0.06051712 \\
\hline 0.05741157 & 0.00376701 & 0.01287843 & 0.00374945 & 0.01493618 & 0.06051712 \\
\hline 0.05741157 & 0.00376701 & 0.01287843 & 0.00374945 & 0.01493618 & 0.06051712 \\
\hline 0.05741157 & 0.00376701 & 0.01287843 & 0.00374945 & 0.01493618 & 0.06051712 \\
\hline
\end{tabular}

Como paso 4, la distancia ideal en la solución es necesario cuantificar. Las dos distancias Euclidianas para cada alternativa se calculan de acuerdo a las ecuaciones (10) y (11). La proximidad relativa se calcula por la ecuación (12). Y el resultad de esto se presenta en la tabla 6.

$$
\begin{gathered}
S_{i}^{+}=\sqrt{\sum_{j=i}^{n}\left(V_{i j-} V_{j}^{-}\right)^{2}} \quad i=1,2, \ldots, n \\
S_{i}^{-}=\sqrt{\sum_{j=i}^{n}\left(V_{i j-} V_{j}^{-}\right)^{2}} \quad i=1,2, \ldots, n \\
C_{i}=\frac{S_{i}^{-}}{S_{i}^{-}-S_{i}^{-}} \quad i=1,2, \ldots, m ; 0 \leq C_{i} \leq 1
\end{gathered}
$$

Tabla 6. Método TOPSIS-Resultados

\begin{tabular}{|l|l|l|l|l|}
\hline \multicolumn{1}{|c|}{ MATERIAL } & \multicolumn{1}{c|}{ Si +} & \multicolumn{1}{c|}{ Si -} & \multicolumn{1}{c|}{ Ci } & \multicolumn{1}{c|}{ RANGO } \\
\hline ACERO GALVANIZADO A36 & 0.02523221 & 0.11270203 & 0.8170707 & 4 \\
\hline ACERO 1000 & 0.03940625 & 0.10418871 & 0.7255736 & 2 \\
\hline $2.5 \% \mathrm{Mn}+97.5 \% \mathrm{Al}$ & 0.11789607 & 0.02638399 & 0.18286649 & 1 \\
\hline ACERO 1045 & 0.00068722 & 0.20747453 & 0.99669863 & 6 \\
\hline ACERO 1020 & 0.01392978 & 0.12678539 & 0.90100727 & 5 \\
\hline ACERO 1006 & 0.02403842 & 0.12410231 & 0.83773255 & 3 \\
\hline
\end{tabular}




\subsection{Método VIKOR}

Según Girubha y Vinodh (2012) el método VIKOR busca aquella solución que esté más cerca de la solución ideal positiva (SI+) y más lejos de la solución ideal negativa (SI). Como primer paso es necesario calcular los mejores, $f_{i}^{+}, y$ los peores, $f_{i}^{-}$, es decir los valores de cada criterio. Como paso 2 , se calculan valores $S_{j}, R_{j} y Q_{j}$, para cada alternativa, de acuerdo a las siguientes ecuaciones $13,14,15$.

$$
\begin{aligned}
& S_{j}=\sum_{i=1}^{n} \quad w_{i} \frac{f_{i}^{+}-f_{i j}}{f_{i}^{+}-f_{i j}} \\
& R_{j}=\max _{i}\left[w_{i} \frac{f_{i}^{+}-f_{i j}}{f_{i}^{+}-f_{i j}}\right] \\
& Q_{j}=v \frac{S_{j}-S^{*}}{S^{-}-S^{*}}+(1-v) \frac{R_{J^{-}-R^{+}}}{R^{-}-R^{+}}
\end{aligned}
$$

Como paso 3, consiste en ordenar las alternativas, según valores $S$, R y Q, en orden decreciente. Se obtiene la tabla 7 como resultado de método VIKOR.

Tabla 7. Método VIKOR-Resultados

\begin{tabular}{|l|l|l|l|l|l|l|}
\hline \multicolumn{1}{|c|}{ Material } & $\begin{array}{c}\text { Magnitud } \\
\text { máxima }\end{array}$ & \multicolumn{2}{|c|}{ Solución ideal positiva } & $\begin{array}{c}\text { Magnitud } \\
\text { mínima }\end{array}$ & Ponderación & Resultado \\
\hline $\begin{array}{l}\text { ACERO } \\
\text { GALVANIZADO A36 }\end{array}$ & F1 Max & 0.13608392 & F1 min & 0 & P4 & 0.16273422 \\
\hline ACERO 1000 & F2 Max & 0.13608392 & F2 min & 0 & P2 & 0.21046025 \\
\hline $\mathbf{2 . 5} \%$ Mn+97.5 \% Al & F3 Max & 0.291 & F3 min & 0 & P1 & 1 \\
\hline ACERO 1045 & F4 Max & 0.13810897 & F4 min & 0 & P6 & 0.00653598 \\
\hline ACERO 1020 & F5 Max & 0.138919 & F5 min & 0 & P5 & 0.15617041 \\
\hline ACERO 1006 & F6 Max & 0.139 & F6 min & 0 & P3 & 0.17205284 \\
\hline
\end{tabular}

\subsection{Discusión}

Según Ashby y Johnson (2010) la característica de un acero dulce no aleado convencional, se encuentra en ser laminado en frío con un bajo porcentaje de carbono, siendo un acero prácticamente suave, por esta razón, deben ser de mayores espesores, para soportar esfuerzos a los que se encuentran sometidos, se emplean como láminas para techar paneles automotrices. Youn (2004), afirma que los aceros de alta resistencia se dividen según el proceso de endurecimiento, es decir, en aceros reforzados, microaleados y refosforados, que se emplean en la industria automotriz en la fabricación de molduras exteriores, como son, puertas, cofres, techo, o elementos estructurales como bastidores inferiores, refuerzos de suspensión, travesaños, largueros.

Chatterjee y Chakraborty (2012) indican la importancia de los materiales en el proceso de diseño de ingeniería para un producto en particular, ya que es una de las tareas críticas para los diseñadores, con el fin de encajar conceptos de diseño factibles y cumplir los requisitos finales 
del producto. Hay una gran variedad de materiales con diversas propiedades disponibles para los diseñadores para satisfacer diferentes requerimientos de diseño. Según el método COPRAS se verifica que el material ideal en la construcción de un habitáculo de seguridad sería el Acero 1045, por las diferentes características mecánicas que posee.

Según Pla (2017) el SIP de cada criterio será el máximo si se quiere maximizar el criterio y el mínimo si se quiere minimizar el criterio, y el NIP será el mínimo si se quiere maximizar el criterio y el máximo si se quiere minimizar el criterio. La tabla 9 indica los resultados de mayor a menor, de los materiales idealizados con mejores prestaciones, utilizados para el presente estudio. Para el Método VIKOR la tabla 8 indica los resultados de los materiales idóneos. Se verifica que un resultado es aceptable cuando se tiene los siguientes parámetros. Y confirma $Q\left(A^{2}\right)-Q\left(A^{1}\right) \geq D Q$, donde $A^{2}$, Es la segunda alternativa según la clasificación de los valores de $\mathrm{Q}$, y $D Q=\frac{1}{J-1}$, siendo j el número de alternativas. Y en el método VIKOR como resultado se obtiene que el acero 1045, sea la mejor opción y sustituto para un habitáculo de seguridad, mientras que la Aleación Aluminio Magnesio, a pesar de ser un material ligero se visualiza que no resistiría fuerzas de impacto.

De acuerdo a Chen et al. (2015), respecto de los tipos de materiales empleados en las carrocerías, el acero ocupa el $75 \%$ del peso total, seguido por aluminio, plásticos entre otros. La tabla 8, indica los diferentes rangos de límite elástico para diferentes tipos de acero empleados en la fabricación de una carrocería en un vehículo.

Tabla 8. Rangos de límite elástico para los diferentes tipos de acero

\begin{tabular}{|l|l|l|l|}
\hline \multicolumn{1}{|c|}{ GRUPO } & \multicolumn{1}{|c|}{ LÍMITE ELÁSTICO (MPa) } & \multicolumn{1}{|c|}{ TIPO } & \multicolumn{1}{c|}{ MANUFACTURA } \\
\hline Aceros convencionales & $<200$ & & \\
\hline Aceros de alta resistencia & $160-300$ & BH & Reforzados en hornos \\
\hline & $>340$ & ALE & Afino de Grano \\
\hline & $>220$ & Reforzado & Solución sólida \\
\hline Aceros de muy alta resistencia & $500-600$ & DP & Fases duras \\
\hline & $600-800$ & TRIP & Fases duras \\
\hline & $800-1000$ & CP & Fases duras \\
\hline Aceros de ultra alta resistencia & $1000-1250$ & MS & Fases duras \\
\hline & $>1250$ & BOR & Fases duras \\
\hline
\end{tabular}

De acuerdo a Benedyk (2010), este indica que el aluminio es ligero en relación con el acero inoxidable, de tal forma que su empleo en la manufactura automotriz, incrementa constantemente, siendo la primera aleación ligera, junto con el Titanio y el Magnesio. Otra característica señala al Aluminio de producción económica, sin embargo, se encuentra en relación 2:1, con respecto a los aceros.

En la tabla 9 se muestra una comparativa de los materiales evaluados por los tres métodos. Se observa que en los tres estudios el mejor material sustituto es el Acero 1045, seguido por el Acero 1020, acero 1006, cabe mencionar que las variables analizadas son las características técnicas de cada material. 
El rango de calificación para el método COPRAS fue de forma descendente, donde 6 es la mejor opción, mientras que en los Métodos TOPSIS y VIKOR, es de forma ascendente, donde 1 es la opción dominante.

Tabla 9. Rangos de límite elástico para los diferentes tipos de acero

\begin{tabular}{|l|l|l|l|l|l|l|}
\hline \multirow{2}{*}{ Material } & \multicolumn{2}{c|}{$\begin{array}{c}\text { Método } \\
\text { COPRAS-Resultados }\end{array}$} & \multicolumn{2}{c|}{$\begin{array}{c}\text { Método } \\
\text { TOPSIS-Resultados }\end{array}$} & \multicolumn{2}{c|}{$\begin{array}{c}\text { Método } \\
\text { VIKOR-Resultados }\end{array}$} \\
\cline { 2 - 8 } & \multicolumn{1}{|c|}{ Puntaje } & Rango & Puntaje & Rango & Puntaje & Rango \\
\hline ACERO GALVANIZADO A36 & 98.2696 & 3 & 0.8170707 & 4 & 0.16273422 & 4 \\
\hline ACERO 1000 & 93.8743 & 2 & 0.7255736 & 2 & 0.21046025 & 2 \\
\hline $2.5 \%$ Mn+97.5 \% Al & 79.8757 & 1 & 0.18286649 & 1 & 1 & 1 \\
\hline ACERO 1045 & 116.2756 & 6 & 0.99669863 & 6 & 0.00653598 & 6 \\
\hline ACERO 1020 & 111.7622 & 5 & 0.90100727 & 5 & 0.15617041 & 5 \\
\hline ACERO 1006 & 100 & 4 & 0.83773255 & 3 & 0.17205284 & 3 \\
\hline
\end{tabular}

\section{Conclusiones y recomendaciones}

La selección del material citado demuestra la aplicabilidad, utilidad y exactitud de un modelo de decisión como son COPRAS, TOPSIS y VIKOR, que tienen un alto potencial en la solución a problemas de selección de material complejo, que implican criterios cualitativos, cuantitativos. En todos los métodos considerados, se consigue una mejor evaluación de los materiales alternativos, es decir, se observa el mejor y peor de los materiales estudiados, así como también, que son los mismos en los tres métodos analizados.

El estudio y la metodología de análisis planteados para la validación del material, que podrá servir de base y objeto de estudio para ensayos virtuales de impactos frontales, laterales y por alcance. Además de proporcionar un documento técnico que posibilite los criterios cualitativos, cuantitativos para aplicaciones de ingeniería maximizando la materia prima local.

\section{Bibliografía}

AGENCIA NACIONAL DE TRÁNSITO. (2015). "Estadísticas de transporte terrestre y seguridad vial" 2015 [En línea] Recuperado: http://www.ant.gob.ec/index.php/ noticias/estadisticas\#.Virc5EqxZER (febrero, 2015)

Arízaga Cáceres, E. R., \& Gómez Rodríguez, D. P. (2015). Análisis de los sistemas faltantes de seguridad vehicular de la categoría M1 en la norma NTE INEN 034: 2010 y una propuesta de mejora (Bachelor's thesis).

Ashby,M. y Johnson, K. (2010). Materials and Design: The Art and Science of Matrial Selection in Product Design, 2. ${ }^{\text {a }}$ ed.. Nueva York: Ed. Elsevier, pp 26-244.

Benedyk, J. C. (2010). Aluminum alloys for lightweight automotive structures. En P. K. Mallich, edit.Materials, Design and Manufacturing for Lightweight Vehicles, 79-113. Nueva York: Hardcover.

Chatterjee, P., \& Chakraborty, S. (2012). Material selection using preferential ranking meth-ods. Materials \& Design, 35: 384-393.

Chen, H., Yang, Y. \& Wang, L. (2015). Vehicle front structure energy absorbing optimization in frontal impact. The Open Mechanical Engineering Journal, 9 (1): 168-172. 
Chérrez-Troya, M., Martínez-Gómez, J., Peralta-Zurita, D. \& Antonio Llanes-Cedeño, E. (2018). Métodos multicriterio aplicados en la selección de un material para discos de freno. Ingenius. Revista de Ciencia y Tecnología, 20: 83-95.

Duan, L., Sun, G., Cui, J., Chen, T., Cheng, A. \& Li, G. (2016). Crashworthiness design of vehicle structure with tailor rolled blank. Structural and Multidisciplinary Optimization, 53 (2): 321-338.

Girubha, R. J., \& Vinodh, S. (2012). Application of fuzzy VIKOR and environmental impact analysis for material selection of an automotive compo-nent. Materials \& Design, 37: 478-486.

Guardia, Y., J. Márquez, V. Sánchez, E.A. Llanes-Cedeño, J.C. Rocha-Hoyos y D.B. Peralta. (2018). Mejoras a la asignatura Diseño Estadístico de Experimentos para estudiantes de la carrera de Ingeniería Mecánica. Revista ESPACIOS, 39 (30).

KundakCı, N., \& Işık, A. (2016). Integration of MA-CBETH and COPRAS methods to select air compressor for a textile company. Decision Science Letters, 5 (3), 381-394.

Llanes-Cedeño, Rocha-Hoyos, Peralta, Martínez, y Celi. (2018). Project-based learning case of study education in automotive mechanical engineering. Revista ESPACIOS, 39 (25).

Mishra, N. K., \& Pradhan, S. K. (2016). Crashwor-thiness of a Truck Cabin using Finite Element Simulation. International Journal of Vehicle Structures \& Systems (IJVSS), 8 (4).

Pla, V. (2017). Aplicación de la toma de decisión multicriterio al diseño sostenible de puentes de hormigón. Máster Universitario en Ingeniería. Politecnica de Valencia. España.

Rocha-Hoyos, J. C., Llanes-Cedeño, E. A., Peralta-Zurita, D. \& Pucha-Tambo, M. (2019). Caracterización mecánica a flexión de materiales compuestos con matriz fotopolimérica reforzados con fibras de abacá y cabuya mediante impresión 3D. Ingenius. Revista de Ciencia y Tecnología, (22): 100112.

Rocha-Hoyos, J., Tipanluisa, L. E., Reina, S. W. \& Ayabaca, C. R. (2017). Evaluación del Sistema de Tracción en un Vehículo Eléctrico Biplaza de Estructura Tubular. Información tecnológica, 28 (2): 29-36.

Vega, W. H., E. A. Llanes-Cedeño, J. V. Molina y J. C. Rocha-Hoyos (2018). Revisión de las Características de Modelado y Optimización para el Diseño del Sistema de Suspensión Macpherson, Inf. Tecnol., 29 (6), 221-233.

Villarruel-Jaramillo, A., Rocha-Hoyos, J., Llanes-Cedeño, E. \& Martínez-Gómez, J. (2019). Modelo computacional para reemplazar el material del refuerzo inferior de parachoques en preprototipado. CienciAmérica, 8 (2): 11-29.

Wang, T., \& Wang, L. (2016, August). Numerical Simulation and Structural Improvement for the Crashworthiness Capacity of M1 Type Commercial Vehicle. In ASME 2016 International De-sign Engineering Technical Conferences and Computers and Information in Engineering Conference (pp. V003T01A004-V003T01A004). American Society of Mechanical Engineers.

Youn, B. D., Choi, K. K., Yang, R. J. \& Gu, L. (2004). Reliability-based design optimization for crashworthiness of vehicle side impact. Structural and Multidisciplinary Optimization, 26 (3-4): 272-283.

Zafra-Cabeza, A., Velarde, P. \& Maestre, J. M. (2019) Multicriteria optimal operation of a microgrid considering risk analysis, renewable resources, and model predictive control. Optimal Control Applications and Methods, 1-13 